\title{
Study Protocol Version Condition Coding System
}

National Cancer Institute

\section{Source}

National Cancer Institute. Study Protocol Version Condition Coding System. NCI

Thesaurus. Code C94112.

The coding system used for recording conditions that are the focus of a study. 